\title{
Canonical partition functions in lattice QCD at finite density and temperature
}

\section{V.G. Bornyakov}

School of Biomedicine, Far Eastern Federal University, Vladivostok 690950, Russia, NRC "Kurchatov Institute" - IHEP, 142281 Protvino and ITEP, Moscow, 117218 Russia E-mail: vborneitep.ru

\section{Boyda*}

School of Biomedicine, Far Eastern Federal University, Vladivostok 690950, Russia E-mail: Boyda_D@mail.ru

\section{V.A. Goy}

School of Biomedicine, Far Eastern Federal University, Vladivostok 690950, Russia E-mail: vovagoy@gmail.com

\section{A. Molochkov}

School of Biomedicine, Far Eastern Federal University, Vladivostok 690950, Russia E-mail: amurg@mail.ru

\section{A. Nakamura ${ }^{\dagger}$}

School of Biomedicine, Far Eastern Federal University, Vladivostok 690950, Russia, RCNP, Osaka University, Osaka, 567-0047, Japan,

Nishina Center, RIKEN, Wako 351-0198, Japan

E-mail: nakamuraean-pan.org

We study the QCD matter at finite density and temperature. The sign problem is overcome by our new cannonical approach. We compare RHIC energy scan data and lattice QCD simulations.

37th International Symposium on Lattice Field Theory - Lattice2019

16-22 June 2019

Wuhan, China

*Present address: Center for Theoretical Physics, Massachusetts Institute of Technology, Cambridge, M 02139, U.S.A.

${ }^{\dagger}$ Speaker. 


\section{Introduction: How can lattice QCD contribute Experimenta at Finite density QCD ?}

Lattice QCD simulations are a non-perturbative study based on the first principle. There have been remarkable progresses in numerical simulations in these twenty years. On the other hand, heavy ion collision experiments have been exploring QCD matter at finite temperature and density.

In rellativistic heavy ion collision experiments, an equilibriated state is expected to appear with the chemical potential $\mu$ and the tempearture, $T$, and we may find a rich QCD phase structure [1]. The state is described by the grand canonical partition function,

$$
Z(\mu, T, V)=\operatorname{Tr} e^{-(H-\mu \hat{N}) / T} .
$$

Higher moments are calculated as

$$
\chi^{n} \equiv\left(T \frac{\partial}{\partial \mu}\right)^{n} \log Z
$$

The parameters, $\mu$ and $T$ are changing as the incident energy varies. $N$ is a conserved charge, such as the baryon number, the electro-magnetic charge or the strengeness.

The lattice QCD simulation can evaluate Eq. 1.2), i.e., $\langle N\rangle$ (for $n=1$ ) and its derivatives. Therefore, the number is a point of the contact of the experiment and the lattice QCD.

\section{Canonical approach}

For many years, the sign problem was an obstaclt for the lattice QCD to study finite densty states. In the path-integral,

$$
Z=\int \mathscr{D} U \operatorname{det} D(\mu) \mid e^{-S_{G}} .
$$

the fermion determinant, $\operatorname{det} D(\mu)$ becomes complex for the finite density, i.e., $\mu \neq 0$.

In order to avoid the sign problem, the canonical approach was proposed[2]. The grand partition function, $Z$ has the following form:

$$
Z(\mu, T)=\sum_{n} Z_{n}(T) \xi^{n}
$$

where $\xi$ is fugacity $\xi \equiv e^{-\mu / T}$. The canonical partition function, $Z_{n}$, can be obtained from $Z(\mu, T)$ in the pure imaginary chemical potential:

$$
Z_{n}=\frac{1}{2 \pi} \int_{-\pi}^{\pi} d \frac{\mu_{I}}{T} Z\left(\mu_{I}\right) e^{i n \mu_{I}}
$$

There is no sign problem in the pure imaginary chemical potential regions, in which odet $D$ is real. This method, however, does not work: it suffers from unstable behevior.

In Ref.[5], a new canonical approach was established:

1. To keep the necessary information, we use the multi-precision calculations, especially in Eq.(2.3). 
2. In order to beat the hidden sign problem appearing in evaluationg $Z_{n}$, we fit the number density, $\langle n\rangle$ by Fourier series.

3.

Now we can study the QCD matter at finite density and temperature, i.e., heavy-ion collision experiments can be studies by the lattice QCD.

\section{Analyses of experimental data with lattice QCD}

Experimental multiplicity, $P_{n}$ is given by

$$
P_{n}=Z_{n} \xi^{n}
$$

where $n$ is the net baryon number $n$. Using the $\mathrm{CP}$ invariance $Z_{n}=Z_{-n}$,

$$
P_{n} / P_{-n}=\xi^{2 n}
$$

Therefore, from experimental data $P_{n}$, one can determine $\xi$. Then we can evaluate also $Z_{n}$ [6].

The method can be used only when data of $P_{n}$ and $P_{-n}$ are available. Alternative approach is that we give $Z_{n}(T)$ by the lattice calculation, and compare experimental data $P_{n}$ with $T$ as a parameter,

$$
\frac{P_{n}}{P_{0}}=\frac{Z_{n}\left(T / T_{c}\right)}{Z_{0}\left(T / T_{c}\right)} \xi^{n}
$$

In Fig1, we show an example. The red stars are experimental data of $P_{n} / P_{0}$ at $\sqrt{s}_{N N}=200 \mathrm{GeV}$. We change $T / T_{c}$ and $\mu / T$ in Eq. (3.3) for determining thee parameters.

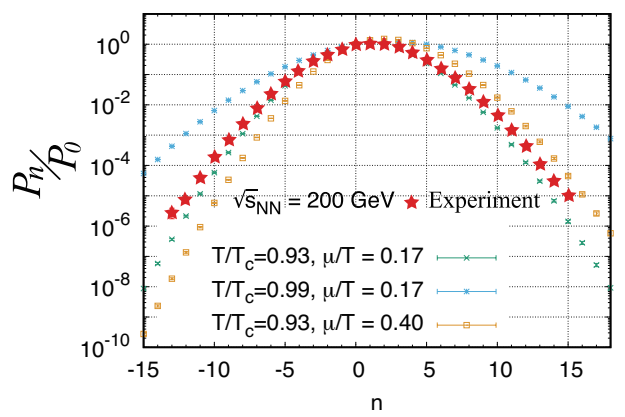

Figure 1: Red star symbols are experimental data. The right hand side of Eq.(3.3) is plotted with $T / T_{c}$ and $\mu / T$ as parameters.

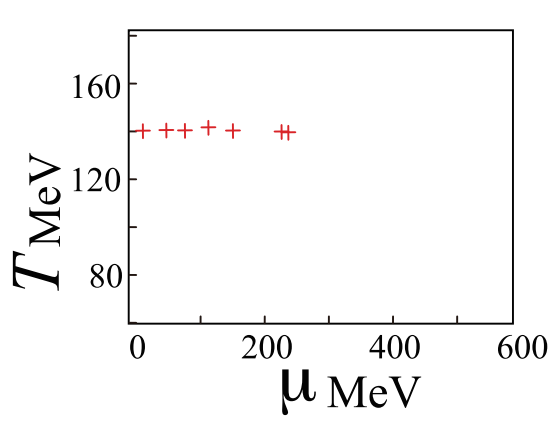

Figure 2: $\mu-T$ regions covered by this analysis. $\sqrt{s}_{N N}=200,62.4,39,27,19.6,11.5,7.7$ $\mathrm{GeV}$ from left to right.

\section{Summary}

In this report, we present our trial to study the QCD matter at finite density and temperature. This is a demonstration to show this new method. Experimental data used here are those of the net-proton, while the lattice data correspond to the net-baryon. 
If experimental data of the electro-magnetic charge, or the strangeness, both experiments and lattice simulations stand for the same quantities.

The work was completed due to support of the Russian Foundation for Basic Research via grant 18-02-40130 mega.

\section{References}

[1] Krishna Rajagopal, 'The Phases of QCD in Heavy Ion Collisions and Compact Stars', 7th Conference. AIP Conference Proceedings, Volume 549, pp. 95-119 (2000). arXiv:hep-ph/0009058

[2] A. Hasenfratz and D. Toussaint, Nucl. Phys. B 371 (1992) 539.

[3] D.Blaschke et al., arXiv:1705.00169 [hep-ph]

[4] J. Cleymans, et al., Phys. Rev. C73 (2006), 034905.

[5] V.G. Bornyakov, D.L. Boyda, V.A. Goy, A.V. Molochkov, Atsushi Nakamura, A.A. Nikolaev, and V.I. Zakharov, Phys. Rev. D95, 094506, 'New approach to canonical partition functions computation in N_f = 2 lattice QCD at finite baryon density', arXiv:1611.04229 [hep-lat]

[6] A. Nakamura and K. Nagata, Prog. Theor. Exp. Phys. 2016, 033D01, 'Probing QCD Phase Structure by Baryon Multiplicity Distribution', arXiv:1305.0760 [hep-ph]

[7] V. A. Goy, V. Bornyakov, D. Boyda, A. Molochkov, A. Nakamura, A. Nikolaev, V. Zakharov, 'Sign problem in finite density lattice QCD', Progress of Theoretical and Experimental Physics, 031 D01. arXiv:1611.08093 [hep-lat]

[8] X. Luo and STAR Collaboration, Journal of Physics: Conference Series 316, 012003 (2011), 1106.2926.

[9] V. Vovchenko, A. Pasztor, Z. Fodor, S. D. Katz, and H. Stoecker, Physics Letters B 775, 71 (2017), arXiv:1708.02852. 